\title{
The fragile and flawed existence of questionable water purity as used in wet chemistry and in particular where such water is destined for medical applications such as vaccines
}

\begin{abstract}
With a constantly escalating and increasing knowledge in both Science and Technology of today, even embracing the World of the Quantum, it is rather surprising and disappointing that wet chemistry as it is related to so-called colloidal silver is not keeping pace with these new developments. In fact little has changed in the last eighty years. Laboratories are still dissolving silver in Nitric acid. This is like practicing alchemy, harking back to the dark ages. Such perpetuation of shared complacency in wet chemistry is evidenced by a complete lack of International Standards for Colloidal Silver and the eventual banning of such materials by Governmental Legislation in a number of Countries, such as The USA (FDA) and Australia (TGA). Being introduced to this wet chemistry in 2008, it was soon realised that Wet Physics and the Photo-electric Effect (explained by Albert Einstein in 1909) made better scientific sense for a new and consistent basis for production. As a direct result, a new material was created without irrelevant chemistry of any kind. Simultaneously it was also realised that the water used as the aqueous media, formed not only an integral part but also the bulk of the material in a ratio of 1 million $\mathrm{mg} /$ litre to $10 \mathrm{mg} /$ litre. Not long after, the purity of water came under scrutiny. Considerable searches in books and on the Internet revealed a large score of anomalies without much of an explanation. Words and descriptions used for measuring current flow in solid metallic conductor were borrowed for measuring purity and other aspects of water, as if metals and water had identical properties. This condition has persisted to this date. In this paper you will be introduced to an entirely new and more direct dual approach for evaluating the purity of water, ensuring an overall quality suited for its intended purpose, i.e. general Injections and vaccines.
\end{abstract}

Keywords: structured and quantum confined water, hydrated electrons, immersed and uncharged organic, inorganic solids
Volume 4 Issue 4 - 2017

\author{
Hans Laroo \\ Independent Researcher, Australia
}

Correspondence: Hans Laroo Independent Researcher, one mile and laroo4305 Australia, Email hlaroo@bigpond.com

Received: May 29, 2017 | Published: August 04, 2017

\section{Introduction}

Life is fickle and what can happen often does, but what about happenings and even misadventures that are difficult or even impossible to detect? Normally tiny anomalies are ignored when their use is non-critical, but when they are important we must be made aware of these. Considering the ubiquitous nature of water forming the carrying agent for medicine and vaccines, anything that can influence the quality of the material must be avoided or at best minimised to an acceptable level. With water able to dissolve almost anything as well as allowing many foreign materials to remain, the risk of contamination becomes very real. To illustrate this point, let us take 'under the loupe' so to speak, the matter of so-called colloidal silver, the subject of my research. Native silver is found as a conglomerate of many other minerals and especially heavy metals such as lead, copper, iron and even arsenic in varying proportions. Depending on how much or how little purification the silver has been subjected to, the ratios of silver and other matter does not change, but their quantities do. Such being the case, highly purified silver at $99.999 \%$ will contain much less of these often hazardous materials than would silver at $99 \%$, and this is the case with medicine and vaccines as well when water is part of the equation. Water is not just water, it is a liquid able to contain just about anything. It is a fact that absolutely pure homogenous water does not exist, perhaps it would, but only in text books. Even when ultra-pure water is produced entirely in a closed system, exposure to air, whatever is in our atmosphere will 'dirty' the water in an instant.
The most common substance to do so will be carbon dioxide. This can be a hindrance or beneficial, depending on its use. Water is a very strange material formed by two atoms of hydrogen (all pervasive in our universe and our stars) and one atom of Oxygen, i.e. $\mathrm{H}_{2} \mathrm{O}$. These are actually two different gases that at normal temperature would be just that: gases, but for some reason have bonded into a liquid like no other and essential for life anywhere here on earth and beyond. Although we have known about water for millennia, researched this liquid, pulled it apart and written many papers and books on the subject, it is still hiding most of its complete identity. Recently a water molecule was discovered inside a crystal of Beryl (element Beryllium) such as an Aquamarine or Emerald. Most likely this was formed billions of years ago. What is so amazing: the water molecule occupied a space smaller than itself. In order to do so, the molecule had managed to stretch itself by lengthening the distance between the three atoms until it could. Something similar must be taking place with capillary action and other intracellular access of water into tight places. Such severe confinement is now referred to as Quantum Confined Water. ${ }^{1}$ Water is capable of much amazing behaviour, such as its structure formation of water molecules. These structures form depending solely on what material it is in contact with or what it is contained in. It has even been endowed with some type of memory by being able to remember which material it last touched, such as homeopathic medicines diluted to such an extent that no trace of the last molecule had remained. ${ }^{2,3}$ Research on these phenomena was done by Madeleine Ennis, a 
pharmacologist at Queens University in Belfast on the substance 'Histamine'. Inflammation Research, vol.53 page 181. Naturally, this is not strictly a 'real memory' but a temporary retention of the last event before new information from a different substance is acquired. Real memory can recall an event as often as desired. As soon as a new material is added, such information changes. Besides of all that, water is known to make and break hydrogen bonds on time scales of $10^{-18}$ seconds. Another peculiar behaviour of water is its cohesion or repelling action known as hydrophilic or hydrophobic tendencies respectively. Incidentally with neutral silver it is hydrophobic but with ionic silver apparently hydrophilic, as this material is totally dissolved in the water. It cannot be observed and only measured in its ionic state, i.e. as a positively charged action. The strangest condition of all is the capture of lone electrons called solvated or hydrated electrons. Their release can only come about by a chance collision with a photon of sufficient energy, understood to be of no less than $2.6 \mathrm{eV}$. Likewise it is claimed that some molecular formations of water can take the shape of a prism, a crate-like structure or something like an open book shape. It is claimed that the first two formations can actually contain materials other than its self. ${ }^{3,4}$ Considering these weird phenomena that this, presumed simple water is capable of, we are reminded by Lao Tsu's comment in Tao Te Ching 'nothing is more soft and yielding than water, yet for attacking the strong, it has no equal".

In 1940, N. Ernest Dorsey, Physicist for the National Bureau of Statistics USA wrote a Monograph Titled: Properties of ordinary WATER-SUBSTANCE in all of its Phases: Water vapor, Water, and all the ices, under the heading of Geo-Physics. It contains 673 pages of absolute technical information. Yet much very relevant information has been left out, such as what is the electrical resistance of water and how to measure this. ${ }^{5}$ A hundred years earlier Michael Faraday, an almost accidental scientist discovered the effect of plane polarized light travelling through water. Under the influence of a magnetic field this light would and travel in a spiralling trajectory. It is called the Faraday Effect and can be used to determine certain properties of water. More recently, in 2013, another book on water titled: The Fourth Phase of Water by Professor Gerald H. Polack was published by Ebner and Sons, Seattle, USA. ${ }^{6}$ What all of these books have in common as well as a number of papers by Anthony Bevilaqua et al, is the total lack of detail on instrumentation used in measuring the electrical properties of water and in particular its electrical resistance to electrical current flow. According to the paper: The Fundamental Conductivity and Resistivity of Water (2005), "at $25^{\circ} \mathrm{C}$ the accepted values with their uncertainties for

\section{$\mathrm{K}($ conductivity $)=0.05501$ micro Siemens, and}

\section{$\mathrm{P}($ resistivity $)=18.18 \mathrm{M} \mathrm{Ohm"}$}

Note! In this study, it is claimed, that whilst Conductivity $(\mathrm{K})$ is determined by way of precise measurement, the value of resistivity (p) is instead calculated, seemingly too difficult to measure such values accurately. In an article carrying the same title but released in 2004, the following comments are noteworthy: 'The resistivity change for impurity levels below one micro gram/L could not be calculated and continuing that this is an unacceptable limitation for modern conductivity instrumentation'. Also missing are references to any existing electrical interfacial charges between water and other matter, such as the static electrical interfacial charge referred to as the Zeta potential and other electrical charges such as the Stern and Nernst potentials respectively. Fortunately, BETZ's Handbook of Industrial Water Conditioning, published in 1980 (second printing in 1982) is more forthcoming in dealing with the electrical properties of water. Nevertheless, really important information as to how to properly measure the electrical properties of water with $\mathrm{pH}$ meters and conductivity meters in particular are yet to materialize. ${ }^{7}$ Wet chemistry places too much reliance on measuring conductivity, as if knowledge of the ionic state of water is all that is required without considering any uncharged material, either organic and/or inorganic substances, also found in water. Conductivity and its reciprocal Resistivity are incomplete, lacking the voltage potential element that has been conveniently left out. It is incomprehensible how conductance measured in micro Siemens and Resistivity, its alleged reciprocal, can be measured over a centimeter distance as though hardly comparable to current over time (ampere/hour) and resistance/ resistivity in Ohms and voltage potential forming part of that equation. This hiatus between conductivity/resistivity over distance instead of current and resistance over time (hour) is a major hurdle for wet chemistry to reconcile with and part of the reason for this paper. The subject is further explained in the following section.

\section{The current technical application of water purity testing}

In the Abstract a dual approach was mentioned and with that is meant to not only determine the electrically charged status of the water, but also the total content of both immersed organic and inorganic material in the water. To determine the electrical opposition of water to current flow, the Conductivity meter is very much 'in vogue' at present. It measures total ionic content dissolved in the water, i.e. total of cations (positively charged cathode ions) and anions (negatively charged anode ions). Cations are attracted to the cathode electrode and anions to the anode electrode respectively. However this can only happen if these electrodes are subjected to a voltage potential across them. However if that voltage potential is an excess of 1.23 volt DC, the water molecules break up into their respective atoms, that of Oxygen and Hydrogen. In that condition the water is said to be polarised, i.e. negatively charged Oxygen and positively charged Hydrogen have separated. A simple reduction in the applied voltage potential to just 1 volt Direct Current would have avoided such a catastrophic happening. However instead of taking such a logical strategy, 'wet chemistry' decided to use Alternating Current instead and in so doing create a bigger problem than what was there in the first place. The reason is simple, the resistance or resistivity of alternating current (provided it consists of pure sinewaves) is called IMPEDANCE and is frequency sensitive, i.e. different frequencies of sine waves create different impedance. Impedance is an alternating current resistance. Instrumentation using either a higher or lower frequency of operation would present a different impedance to the water to be tested and the readings of instrumentation operating at different frequencies/wavelength cannot possibly compared. However if that was the only problem. We have now learned that Conductivity is now measured using alternating current (electron or charge carrier) flow. But it is simultaneously claimed that resistance or resistivity is inversely proportional to conductance, but it can only be measured in Ohms, a direct current component. Most likely the assumed reciprocal values between conductance and resistance have just been calculated as if it were similar to Ohms Law, but not really ever verified if this was a true state of affairs. Normally conductance and resistance is more accurate when placing the two electrodes of the measuring probe $10 \mathrm{~mm}$ apart and making each electrode $10 \times 10 \mathrm{~mm}$ in size. That creates some sort of standard that is commonly referred to and adopted to conductance AND resistance as resistivity. Both conductance and its reciprocal are both measured in centimetres, i.e. conductance/cm and resistivity in $/ \mathrm{cm}$. Note! Resistivity is a deviation 
from Ohmic resistance and relates to calculating current over a very specific distance, calculated as current flow on te outside of a solid metal conductor cubic in shape and this current being measured at two opposing surfaces of such a cubic form. The first introduction was based on a metre cubic, i.e. 1metre $\mathrm{x} 1$ metre $\mathrm{x} 1$ metre. Incidentally it had nothing whatsoever to do with current flow in water. But more about that later.

Al well-known organization has gone through the process to create a visual representation of the Conductance concept and its reciprocal identity of resistivity and ranges from 1 Siemens $=1 \mathrm{Ohm}$ at extremely high conductivity end to the lowest conductivity/highest resistivity of 0.01 micro Siemens and 100million Ohm respectively at the other extreme as shown hereunder (Table1): The original chart published, runs horizontally and the divisions are all of equal size and thus show a linear relationship, yet within each linear division, the subdivisions indicate a non-linear progress. No mention is made why this is represented in this fashion nor the absence of a voltage potential..$^{8-11}$ In order to lend substance to the claim that the concept of conductance is flawed, I measured Conductance with a conductance meter. Deionised water measured 0.22 micro Siemens and distilled water as 2 micro Siemens. That is about eight times higher in conductivity. Using a variety of analogue and digital multimeters, including a VTVM (stands for vacuum tube voltmeter) with a maximum resistance meter of 1,000million $\mathrm{Ohm}$ and one digital multimeter able to measure up to 200million Ohm resistance, all that these meters could measure was about 3million Ohm for the deionised water and 100,000 Ohm for the distilled water, an obvious discrepancy of at least $90 \%$ error factor. 1micro Siemens conductance should equal a resistivity of 1 million Ohm, yet time and time again, I can only measure $1 / 10^{\text {th }}$ of that value, i.e. $100,000 \mathrm{Ohm}$. These measurements were repeated several times and showed great consistencies over time. Obviously something is seriously wrong. Most likely not taking into account the voltage potential so necessary under Ohm's Law. Contrary to the chart above referring to pure and ultra-pure water, is the information supplied by The European Agency for the Evaluation of Medicinal products (evaluation of medicines for human use) sets the following recommendations for three different qualities of water as:

\section{i. Purified water (PW).}

ii. Highly purified water (HPW). Came into effect in January 1, 2002.

\section{iii. Water for Injection (WFI)}

Table I Chart of micro Siemens as conductivity, resistivity and micro current levels

\begin{tabular}{|c|c|c|c|}
\hline 0.01 micro Siemens $\mathrm{A} / \mathrm{h}$ & 100 million Ohm & (ultra-pure water & 0.1 micro \\
\hline 0.I micro Siemens A/h & 10 million Ohm & (pure water, injections) & I micro \\
\hline I micro Siemens A/h & I million Ohm & (distilled water) & 10 micro \\
\hline I0 micro Siemens A/h & $100,000 \mathrm{Ohm}$ & (rain water) & 100 micro \\
\hline 100 micro Siemens $\mathrm{A} / \mathrm{h}$ & $10,000 \mathrm{Ohm}$ & (tap and boiler water, mineral water) & I \\
\hline I mili Siemens mA/h & I000Ohm & (saline solution, beer and fruit drinks) & $\mathrm{I} \mathrm{mA} / \mathrm{h}$ \\
\hline I0 mili Siemens $\mathrm{mA} / \mathrm{h}$ & $100 \mathrm{Ohm}$ & (sea water) & $10 \mathrm{~mA} / \mathrm{h}$ \\
\hline I00 mili Siemens mA/h & $10 \mathrm{Ohm}$ & (inorganic acids, strong electrolytes) & $100 \mathrm{~mA} / \mathrm{h}$ \\
\hline I Siemens & I ohm & & $\mathrm{IA} / \mathrm{h}$ \\
\hline
\end{tabular}

Refer: The European Pharmacopoeia 4 ed. 2002. Using conductivity as per the chart appears in order, until we come to the conclusion that conductivity and resistivity without a voltage potential makes no sense. We must thus conclude that the concept of conductivity as such is a mere artefact and a scientific anomaly. More substance to these claims are presented by the following scientific facts:

1. Non-adherence to the well-established OHM's Law in Commerce, Industry and Technical Science in use all over the world wherever electrical and electronic technology is used.

2. It is electrical current that should be measured according to the accepted standard of Ampere or fractions thereof over time, i.e. ampere/hour. Conductance does not even come into that.

3. Current flow or the flow of charge carriers (ionic material moving either toward the anode or cathode) does not and cannot flow in a straight line (linea recta) through water. Also take note that water should never be compared with a solid metal conductor. Water is a known dielectric material and far from being homogenous due to being able to dissolve almost anything as well has hold material in immersed suspension. Electrical current simply needs to flow around all of these obstacles. There is also the constant change in the structure of water molecules themselves as well as the influence of the so-called ever-present Brownian movement.

OHM's Law as related to electrical current and resistance, mentioned earlier, can be visualised being part of a triangle. The voltage potential represented by the letter V located at the tip or apex, and the elements of current (I) and resistance (R) at each corner at the base of the triangle (Figure 1).

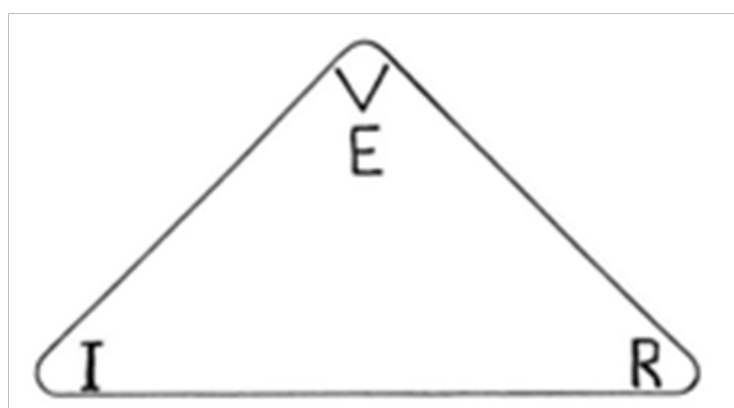

Figure I The well-known triangle representation of the current, resistance and voltage potential relationship. Note! $\mathrm{V}$ for Voltage potential can also be expressed as $E$, so $E=I \times R$

$\mathrm{I}=$ Current, $\mathrm{R}=$ Resistance in OHMS, $\mathrm{V}$ or $\mathrm{E}=$ Voltage potential $\mathrm{I}=\mathrm{V} / \mathrm{R} ; \mathrm{R}=\mathrm{V} / \mathrm{I} ; \mathrm{V}=\mathrm{I} \times \mathrm{R}$

Know the value of two elements and you can calculate the third, i.e. 1 ampere at 1 volt and at $1 \mathrm{Ohm}$, means that at a voltage of 1volt, 1 ampere can flow through a resistor of $1 \mathrm{Ohm}$. Thus if the resistance is halved to $0.5 \mathrm{Ohm}$, twice as much current can flow, i.e. 2 ampere. Subsequently doubling the resistance to $2 \mathrm{Ohm}$ will reduce the current to half that value, i.e. $0.5 \mathrm{Ohm}$ or $500 \mathrm{~mA}$. Note! When according Ohm's Law, 1Ohm equals 1 volt at a current of 1 ampere/h, then 1 micro Siemens at 1 million ohm and 1 micro ampere/h, the equivalent voltage potential would be 1 micro volt DC. That is 1 millionth of a volt $\left(10^{-6}\right)$. 


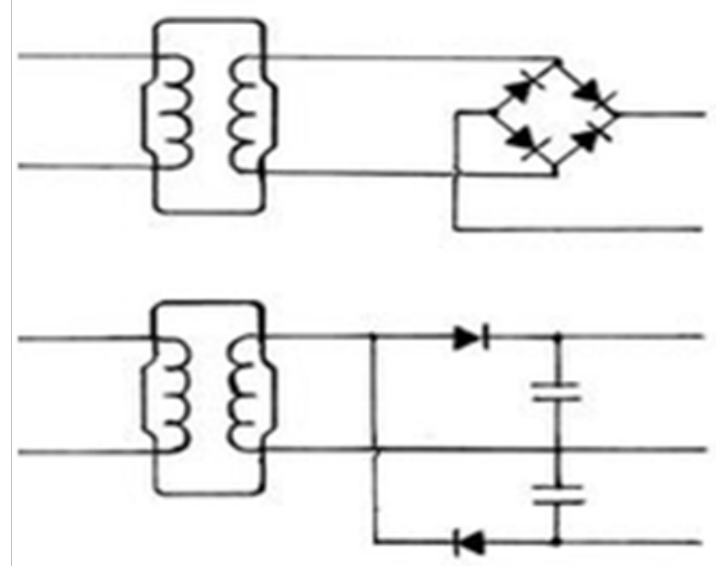

Figure 2 Upper circuit diagram is a full wave rectifier circuit, providing a direct current voltage from a step-downed alternating voltage times I.4, i.e. 24 volt $A C \times 1.4$ equals 33.6 volt DC. The lower circuit diagram is called a voltage doubler circuit, changing the 24 volt $A C$ into two voltages. The mid rail will be at a potential of 33.6 volt DC, the bottom rail is zero volts and the top rail 67.2 volts $D C(33.6 \times 2=67.2)$. The two upright rectangular shapes represent transformers wired for 240 volts $A C$ at the left and 24 volts $A C$ at the right.

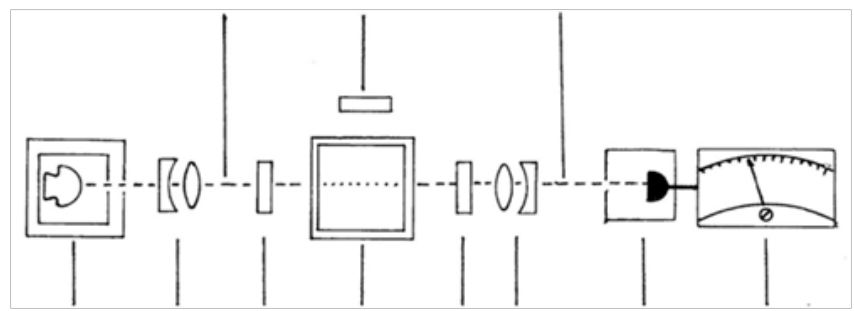

Figure 3 This a schematic representation of a cross polarisation instrument capable of detecting both turbidity (if any) as well as either unpolarised or cross polarised light scattering. In the cross polarised position, metals remain visible and can be recorded, but any material with a refractive index at visible light will be extinguished. A panel meter will quantify any of these two conditions.

Change the voltage and again current and voltage will also change. Usually an increase of voltage will reduce current and a reduction of voltage will increase current flow. It should now become obvious how important knowing the voltage potential is in measuring current flow, something that is totalling lacking in the conductivity/resistivity usage. Why concepts such as Conductivity, Siemens and Resistivity were ever used for measuring electrical current flow in water, makes no logical sense. Siemens, Resistivity and Current have their roots in the physics of solid metal conduction. The Siemens was coined by Werner von Siemens, a German inventor, he used the term to denote a particular resistance of $0.95 \mathrm{Ohm}$ (a narrow column of Mercury), representing a one metre length of telegraph cable in the late 1800's. The term resistivity was also solely dedicated to represent a specific current flow over the surface of a solid metal cubic block of 1 metre cubic, i.e. the number of electrons flowing across it surface between two opposite surfaces over a period of 1 hour. To start using the term Mho as a reverse spelling of Ohm (not as an inverse property as it should) and later twisting it away with the term Conductivity to measure water's opposition to current flow, is simply too fanciful. In order to overcome these anomalies, something more consistent with Physics and more precise will be required. An experiment a few years ago proved also that volume is a relevant factor in determining the resistance of water. ${ }^{8-10}$ During an experiment with a $1,200 \mathrm{~mm}$ long tank, containing 40litres and two silver electrodes placed $1,000 \mathrm{~mm}$ apart, I was able to measure $1,000 \mathrm{Ohm}(1 \mathrm{Giga} \mathrm{Ohm})$ and confirm this reading with an actual $1 \%$ accurate resistor of that same value of $1,000 \mathrm{Ohm}$. In order to compare this test as if it were a conductivity test, a special probe was designed and constructed with the thin wavers of silver measuring $10 \times 10 \mathrm{~mm}$, facing each other at $10 \mathrm{~mm}$ apart and found the resistance to be 300 million ohm. The water was of a deionised type. Only analogue electronics based equipment performed well, but digital multimeters could not cope and even differed amongst themselves. To also prove that the quantity of water (in a larger tank) would increase the resistance and that increasing the voltage potential would reduce that was conducted immediately thereafter. It was started with a low voltage potential of 33.6volt DC, obtained by rectifying a common $\mathrm{AC}$ voltage supply of $24 \mathrm{volt}$. When rectifying such an $\mathrm{AC}$ voltage to $\mathrm{DC}$ and using a smoothing capacitor that formula times 1.4 is used to calculate the actual De voltage obtained, i.e. 24 × $1.4=33.6$ volt DC. Instead of a full wave rectifier it is also possible with a pair of diodes and two capacitors to obtain double the voltage at around 67.2volts DC. These two procedures are referred to as a full wave rectification and a voltage doubler circuit configuration. The advantage of the voltage doubler circuit is that both a DC voltage of 67.2 but also half of that voltage potential is obtained, i.e.33.6volt. Obviously a linear response at such low voltages (Figure 2).

\section{Measuring current through water using DC}

A proper direct current based conductivity meter based on analogue principles has been designed and a prototype built. It boasts an input resistance factor of 10,000 million $\mathrm{Ohm}$ at a DC voltage potential of just 1 volt. It is incapable of polarising the water in any significant way as well as not loading the water with a lower resistance. The parallel resistance factor would cause large error factor (explained in more detail later). It has now been in use for a number of years, operates in a very consistent manner, any time of the year and provides a read-out in less than a second.

\section{The use of optical light scattering and light transmission absorption}

Realising that in any medical injection and vaccines only the tiniest amount of water is used, it nevertheless opens up the means to inspect both the water and content, using static light scattering. There is also dynamic light scattering but this method is not discussed here. The difference between the static and dynamic light scattering techniques can be explained using the analogy of amplitude modulation (AM) in static light scattering and frequency modulation (FM) in dynamic light scattering, respectively. Such static light scattering will pick much detail invisible to the naked eye or even aided with a microscope without the benefit of light scattering. As pointed our earlier, water has peculiar properties, still not well understood, requiring further research and be more carefully scrutinised as to its actual inorganic and organic content in a way different to determination of ionic content. That can be done by measuring the turbidity and the way different wavelengths of light are scattered. Instruments precise enough are actually able to observe interfering phase differences reflected back from larger particles where the reflected or scattered light from one area lags behind light from another area over time. A bit like hearing a difference in the public address messages at a railway platform with speakers placed at intervals. These scientific light scattering instruments are of two basic types and widely available in the 1900s, but have gradually been phased out. They are the nepholometer for light scattering and the sedimentometer turbidity meter in order to 
measure light absorption, indicating contaminants. Research is ongoing to bring modern versions of both types of instruments, preferable combined into one as a means to recognise and quantify the amount of contamination of both an organic and inorganic material. See a graphic illustration of such an instrument below: (Figure 3). Incident light source lens Polariser cell and contents cross polariser lens Photo detector/diode Panel Meter \& scattered light.

\section{Light scattering techniques for identifying and quantifying materials other than water}

Note! Light scattering is no more than reflection of incident light from three dimensional objects, generally at an angle or even different angles when it is ricocheted between these objects. The need for determining contamination of water can to some extent be observed and recorded with optical microscopes in situ, e.g. in water or through the use of electron and scanning electron microscopes, albeit in a dry condition. The problem with that is clumping of materials during the water evaporation process and other airborne materials adding to the sample under test. A viable alternative to these optical and electronic strategies is the use of Static Light Scattering. There is also dynamic light scattering, also known as Photon Correlation spectroscopy and Quasi-elastic light scattering. As stated earlier, for the purpose of the paper only static light scattering will be described. Using static light scattering can be relatively simple or complex as one wishes. The simplest option is to use an LED torch with a single white light source that can be focused near or at a specific distance in the sample. Shining light through the sample will quickly show light being scattered, especially in a darkened room. A red or green laser pointer is even better. A further improvement can be made by different colour light sources, since very small particles of organic or inorganic material would reject long wavelengths due to being unable to scatter a wavelength longer than its own size, except in the case of nano silver where a Local Plasmon Resonance may be created instead. When objects in the water are very small it is recommended to use a light source near the edge of visibility e.g. violet at $405 \mathrm{~nm}$. Light scattering can be substantially enhanced through the use of a pair of linear polarising filters, such as used with $20^{\text {th }}$ Century with analogue cameras. By using one of the polarizing filters as a polariser of the incident light and the other as the analyser, changing one filter's orientation by 90 degrees can actually remove light scattering caused by materials with refractive indexes at wavelengths of visible light. A bit like "now you see it and now you don't" like a well-known parlour trick', except it is not a trick but an interesting scientific phenomena. It works this way. Consider a large shopfront glass window during daylight hours. Ambient light will be reflected from the glass to some extent, even images as if it were a mirror and only part of the incident light will be transmitted through the glass and illuminating objects behind it. Glass having a high refractive index (bending of the light), will make it difficult for observing the items behind the glass or even photographing it until we polarise the light with a linear polarising filter. Something similar can be done making fish in a pond more visible, since water, like glass also has a high refractive index. Think of a pencil in a glass of water. This ability to distinguish materials with or without an obvious refractive index with the use of linear polarising filtering 'Cross Polarised' is an enormous augmentation in this process. It creates an opportunity by way of optical measurement to quantify the differences between material with a refractive index at visible light and those materials that do not, such as Silver and Aluminium that reflect such incident light at very high percentages, i.e. silver at $97 \%$. It enables quantifying such results. Light scattering techniques can be further enhanced with differing wavelengths of light either with specific pass filters or high quality diffraction gratings that provide wide band applications. A few years ago this advantage was proven when a visiting scientist used both a red as well as a green laser diode pointer to scatter light in a number of samples with success. With one sample however, no scatter of light was observable, until I pointed out that the material in there was only in the one digit nanometre range and red and green light was just too long in wavelength to have any effect. Using a blue laser diode at $405 \mathrm{~nm}$ (violet light) showed intense scatter. The reason for mentioning this is that some contaminations can be very small indeed and still prove to be of concern..$^{12,13}$

\section{Using the likes of a Sediment meter or turbidity meter to determine extent of difficult to see contaminations}

In contrast to light scattering techniques, measuring opaqueness of unwanted materials such as dissolved gases like Carbon dioxides and Ammonia. Accurate measurement on the level of incident light traversing through a cell containing a fluid has been used extensively during the 20th Century, but like the nepholometer has also disappeared. Fortunately an updated version with modern technology including laser diodes as transmitters of light and photo diodes as sensitive receivers will make the old incandescent globes quickly redundant. There was too much heat associated with such technologies and often overheated the samples under examination. It is envisaged that two samples should be available. One of which one will be a control sample and the other containing material that may cause a degree of obscurity and thus blocking part or all of the incident light travelling through the cell. The aim will be to have the residual light reaching a photocell or photo diode for quantifying the degree of obscurity. Quantities of water in the production of many vaccines is generally very small, but even so the need for an almost absolute purity and quality of water used must not under-estimated.

\section{Summary and other aspects of water considered relevant in understanding its use as a basis for vaccines and other medications for injection}

Water is finicky and whatever it can contain is most likely to have a deleterious effect on any vaccine, no matter how insignificant it appears. Wherever possible we should make all attempts to get the purest water possible. Not only that we must assure constantly that the water in use is what we expect it to be. Ambient conditions must be looked at that may cause deterioration. This could also be caused by containers leaching out unwanted chemicals or introduced accidentally into the container. On charts seen so far, the purity of water suitable for injections ranges from 1 micro Siemens to 0.1 micro Siemens. That is hardly conducive as a proper standard. As stated earlier, the European Agency for the Evaluation of Medicinal Products divides pure water between Purified Water (PW), Highly Purified Water (HPW) and Water for Injection (WFI). This is not consistent with the norm in use, i.e. Pure water and Ultra-pure water as used in the electronic semiconductor industry at a value of $0.0584 \mathrm{micro}$ Siemens, claimed to be equal to 18.24Million $\mathrm{Ohm}$ at a $\mathrm{pH}$ of 7 at $25^{\circ} \mathrm{Centigrade}$. Considering our International arena of similar industries world-wide all competing the introduction of some standard would be a prudent undertaking. A 1988 chart on the purity of water named Minimum Containment Levels in Highly Purified Water to be expressed as follows: ISO 3696 (187) in purity reducing values as: Grade 1, 2, and 3. ASTM (D1193-91) in purity reducing values as: Type I, II, III and IV. NCCLS (1988) in purity reducing values as: Type I, II and III Pharmacopoeia in purity reducing values as: EP and USP Note! There are 14 separate sections within each group but not shown here. 


\section{Other properties of water to be considered, especially its electrical properties}

There is much more to water than meets the eye, literally. Just listing some of the properties of water relevant to vaccines and injections generally are the: Iso-electric point, $\mathrm{pH}$ factor (alkaline or acidic), Zeta potential, Dielectric properties, Adsorption (water and contents clinging to the walls of a pipe and better flow in the centre of the pipe, osmosis and reverse osmosis, electrical resistance to electrical current flow, generally more difficult as water becomes purer. Water as super-heated steam actually carries static electric charges, and finally quantum confined water. Zeta potential, $\mathrm{pH}$ and the Isoelectric point are nicely placed together on the following diagram, and relevant explanation, kindly with permission from Colloidal Science Laboratories in the US. In Physics and in particular quantum physics, metals such as silver in particular at sizes below $10 \mathrm{~nm}$, Zeta potential takes on a new meaning or perhaps something similar but different but still a very specific static electrical repelling charge. The extent of this repelling (a negative charge for most metals) is not dependent on $\mathrm{pH}$ but on concentration instead, with the metals having been placed in a suspension and not a dispersion. It is further considered that the smaller the size of the nanometre sized material which includes an ever increasing surface area to volume ratio, the higher will be the Zeta potential and its stability, provided the concentration in $\mathrm{ppm} / \mathrm{mg} / \mathrm{l}$ is not greater than allowed by the repelling suspension of individual atomic clusters. It should be noted that this Physics aspect is also applicable to any metals in vaccines. I have produced samples of nanometre sized atomic silver clusters in suspension in 2009 without any obvious change.

\section{The dielectric properties of water, with or without} contaminants

There is no such thing as completely pure water, it may be close but will not stay that way when exposed to the atmosphere even for a second. Scientists involved in the manufacture of ultra-pure water for the semi-conductor industry, use closed systems in its manufacture so that the material is not exposed to air. Nevertheless they report that even under such tight conditions, they still measure some residual conduction of charge carriers, albeit in minuscule amounts. ${ }^{14-16}$ Dielectric properties are all about how insulated or conductive water is to current flow. The fewer contaminants the more it will oppose the flow of electrical current. It is here that the problems arise when trying to measure such minute values. It is caused by an Ohm's Law element referred to as "Parallel Resistance".Ohm's Law states that two resistances in parallel will be less than their individual value and when connected in series will be more than their individual value, i.e. two resistors of $1,000 \mathrm{Ohm}$ each will measure only $500 \mathrm{Ohm}$ when joined together at both ends (parallel) and measure $2,000 \mathrm{Ohm}$ when connected end to end (series). This law has serious implications when measuring the dielectric properties of water and or the allowable current flow. It necessitates using measuring devices and instrumentation with input resistances (Direct Current) or input impedances (Alternating Current) to be considerably higher than that of the water to be measured in order to minimise errors. Such errors can be substantial when the input resistance (DC) or impedance (AC) of an instrument or device is similar to or less than that of water, e.g. If the water has a theoretical resistance of 1 million Ohm for a conductivity reciprocal of 1 micro Siemens and the test instrument or device an identical resistance, than it would only measure half of the value. That is an error factor of $50 \%$. Substantially increasing such input resistances or impedances would minimise the error factor and the measurement would be correct within a few percent. During the 20th Century, the era of Black and White TVs and stereo sound systems, it was considered prudent that in order to not have big error margins to have input resistance at least 10times higher than the maximum resistance encountered of 10 million $\mathrm{Ohm}$. To ensure this could be comfortably accommodated, the Vacuum Tube Voltmeter (VTVM) was designed and placed into operation for technicians to use. It boasted a maximum input resistance of 1,000 million $\mathrm{Ohm}$. That is a 100 times higher and providing a very accurate measurement of anything tested. However a second problem was also underestimated, the equilibrium voltage of water. The equilibrium voltage of water is set at 1.23 volt DC and the voltage potential where water molecules split up into their constituent components of Oxygen and Hydrogen gases. This causes water to polarise, ions being created, and causing an element of uncertainty in measurements. It is recommended that all instrumentation for water testing answer to the requirements of operating on a voltage LESS than 1.23 volt DC and having an input resistance of at least 10,000million Ohm, In order to avoid such problems. The same would apply to current measurements. Perhaps some will think that this seems extreme, but nothing could be further from reality. ${ }^{17-22}$

\section{Conclusion}

It requires stressing the importance that the purity of medical substances in vaccines and the water that is its basis, be always worthy of serious consideration. We should not adopt a complacency seen in a set of instructions for farmers to use "clean water" for their 'on the spot' vaccine preparations without specifying what is meant by clean water. Such a vague term can literally mean anything. The instructions even suggest not to use hot water as this may destroy the contents of the vaccine. No longer should those producing vaccines be satisfied having to deal with references as pure water ranging between 1 and 0.1 micro Siemens, but instead have a guide to a precise determination, i.e. no less than 0.1 micro Siemens or some value in that area that is commercially procurable. "The time has come" the walrus said. To talk of many things (from Lewis Carrol's Through the looking glass), but obviously not having accurate water testing equipment in mind and especially about actual current and resistance in water. So far science has been 'pussy footing around' precise expression as to what actual current and resistances are involved. It is all talk, but no detail. I have searched for some technical references on water measurements besides conductance in micro Siemens, but found absolutely NOTHING! The irony about all this is the fact that the technology for more precise measurements has existed for at least eighty or so years for measuring these higher resistances and lower current levels with substantial improvements in the 1970's by both RCA (Radio Corporation of America) and National Semiconductors. However it required precision to produce instrument designs capable of measuring current at $10^{-12}$ and resistance in $10^{+12}$, in pico ampere $/ \mathrm{h}$ and Tera Ohm levels respectively. It is obvious that improvements in setting minimum and maximum standards are set in place, as well as the introduction of necessary protocols for maintaining such standards.

\section{Acknowledgments}

None.

\section{Conflicts of interest}

Author declares there are no conflicts of interest.

\section{Funding}

None. 


\section{References}

1. Boris P Gorshunov, Elena S Zhukova, Victor I Torgashev, et al. Quantum Behaviour of Water Molecules Confined to Nano cavities in Gemstones. J Phys Chem Lett. 2013;4 (12):2015-2020.

2. Martin Chaplin Water Structure and Science, a series of technical/ scientific papers published on the Internet, Southbank University, London.

3. Cristobal Perez, Matt T Muckle, Daniel P Zaleski, et al. Structures of Cage, Prism, and Book Isomers of Water Hexamer from Broadband Rotational Spectroscopy. Science. 2012; 336(6083):897-901.

4. Findings on how electrons are solvated in water widen the range of potential influences on chemical reactions.

5. N Ernest Dorsey. Properties of Ordinary Water-Substance in all its Phases, Water-vapour, Water and all the ices. Reinhold Publishing Corporation, New York, USA. 1940.

6. Gerald G Pollack. The Fourth Phase of Water (Beyond Solid, Liquid and Vapour). A Hard Copy Book published by Ebner and Sons Seattle, Washington State, USA. 2013. p.357.

7. Inc BETZ Laboratories. Handbook of Industrial Water. Pennsylvania, USA. 1980.

8. Thornton. Ultrapure Water -The Standard for Resistivity Measurements of Ultrapure Water. Massachusetts, USA. 1998.

9. Anthony Bevilacqua, Chemist, Moras Thornton KR, et al. Measurements of the resistivity of Ultrapure Water at elevated temperatures. Ultrapure Water Journal. 1994;1-13.

10. Anthony Bevilacqua, Stuart Licht, Kenneth R Morash, et al. The Fundamental Conductivity and Resistivity of Water. 2005;8(1):E16E19.
11. SIEMENS under "What is a Siemens?".

12. Zygmunt Gryczynski. Depolarized light scattering from silver nanoparticles. 2017.

13. Glauco R Souza, J Houston Miller. Elastic Light Scattering of Biopolymer/Gold Nanoparticles Fractal Aggregates. Reviews in Plasmonics. 2010;39-68.

14. Robert J Hunter. Zeta potential in colloid science: (1st edn). principles and applications. Academic Press, London. 1981.

15. Malmberg CG, Maryott AA. Dielectric Constant of Water from $0^{0}$ to $100^{\circ}$ Centigrade. Journal of Research of the National Bureau of Standards. 1956;56(1)2641

16. Aglaia Vassilikou-Dova, Ioannis M Kalogras, Dielectric Analysis (DEA). John Wiley \& Sons, USA.

17. N Ernest Dorsey. Properties of Ordinary Water-Substance in all its Phases, Water-vapour, Water and all the ices., Reinhold Publishing Corporation, New York, USA. 1940.

18. John F Robyt, Bernard J White. Biochemical Techniques, Theory and Practice, Brooks/Cole Publishing Company, California, USA. 1990.

19. Jesse R Conner. Chemical Fixation and Solidification of Hazardous Wastes, Van Nostrand Reinhold, New York. 1990. p.692.

20. Chen CS, Chung WJ, Hsu IC, et al. Force field measurements within the exclusion Zone of water. J Biol Phys. 2012;38(1):113-121.

21. Anders Nilsson, Lars GM Pettersson. The structural origin of anomalous properties of liquid water. Nature Communications. 2015.

22. Vlad P Sokhan, Andrew P Jones, Flaviu S Cipcigan, et al Signature properties of water: Their molecular origins. PNAS. 2015;112(20):6341-6346. 